\title{
COLONIZAÇÃO ICTIOFAUNÍSTICA DO RESERVATÓRIO DE ITAIPU E ÁREAS ADJACENTES
}

\author{
Evanilde Benedito Cecilio ${ }^{1}$ \\ Angelo Antonio Agostinho ${ }^{1,2}$ \\ Horácio Ferreira Júlio Jr. ${ }^{1}$ \\ Carla Simone Pavanelli ${ }^{1}$
}

\begin{abstract}
ICHTHYOFAUNISTIC COLONIZATION OF THE ITAIPU RESER VOIR AND VICINITY AREAS. Samples were taken from November 1983 to October 1989 in sites along the main body of the Itaipu reservoir and its area of influence to evaluate the impacts of this reservoir on ichthyofauna and to provide information about the colonization process in the dammed area. The ichthyofaunistic similarity between the sites was analysed during the studied period. Spatial and temporal variations of the ichthyofaunistic diversity were also done, using Shannon-Winer Index of Diversity, eveness and species richness, comparing the results with those obtained before the damming. After Itaipu Reservoir formation, it was observed significative alteration in specific abundance and ichthyofauna structure of the Parana River, with an increase in species number in all tributaries of the reservoir, and a high replacement in species. Diversity and species richness were higher in the dammed stretch of the Paraná river and in its tributaries of the left side after the Itaipu reservoir formation. Among the sampled sites, the highest values of the diversity index were registered in Parana River upstream and downstream the reservoir, Iguatemi river and Arroio Guaçu. Based on information of similarity in species composition and abundance in the sampled area, five site groups could be identified.
\end{abstract}

KEY WORDS. Ichthyofauna, Paraná river, Itaipu reservoir, diversity, similarity

A construção de barragens, interceptando cursos de água naturais, têm aumentado de forma significativa nos últimos anos. Estes empreendimentos induzem respostas ambientais complexas, como modificações dos atributos físicos, químicos e biológicos, os quais podem ter seu curso alterado por interações entre estes atributos ou por novas perturbações impostas ao sistema. É improvável a quantificação ou predição concisa destas alterações isoladamente. WETZEL (1990) afirma que, em reservatórios, as respostas naturais, bem como aquelas sob interferência antropogênica, são incompletas, uma vez que são alteradas ou destruídas antes de sua completa efetivação. $\mathrm{O}$ resultado é um incremento caótico na sucessão de respostas, redução na interdependência e menor estabilidade biótica.

1) Departamento de Biologia, Nupélia, Universidade Estadual de Maringá. Avenida Colombo 5790, 87020-900 Maringá, Paraná, Brasil.

2) Pesquisador do CNPq. 
O componente íctico do sistema sofre, nesse processo, profundas alterações. Segundo MERONA (1986), a modificação dos habitats influencia diretamente as funções biológicas de cada espécie de peixe (alimentação, reprodução, crescimento).

A bacia do rio Paraná, a segunda da América do Sul, tem experimentado crescentes reduções de seus trechos lóticos. O rio Paraná, com uma extensão de $810 \mathrm{~km}$ em terrritório brasileiro, tem seu trecho lótico restrito, após o fechamento da barragem de Porto Primavera, a cerca de $230 \mathrm{~km}$ (AGOSTINHO et al. 1995). O reservatório da hidrelétrica de Itaipu, objeto deste estudo, foi formado em 1982 e tem uma extensão de $151 \mathrm{~km}$ e área de $1350 \mathrm{~km}^{2}$.

O presente trabalho analisa as alterações na diversidade ictiofaunística decorrentes da formação do reservatório de Itaipu, as variações espaço-temporais na composição específica e a diversidade durante o processo de colonização do novo ambiente a partir da fauna fluvial.

\section{MATERIAL E MÉTODOS}

Foram estabelecidas onze estações de amostragem, sendo três delas situadas no corpo principal do reservatório de Itaipu na zona fluvial (próximos aos municípios de Guaíra - GUAI), zona de transição (Santa Helena - SHEL) e zona lacustre (Foz do Iguaçu - FOZ), quatro em tributários da margem esquerda do reservatório (rios Ocoí - OCOÍ, São Francisco Falso - SFFA, São Francisco Verdadeiro - SFVE e Arroio Guaçu - GUAC), duas no rio Paraná (uma a montante - MONT e outra a jusante - JUSA do reservatório de Itaipu) e duas em tributários do rio Paraná, a montante do reservatório (rios Iguatemi - IGUA e Piquiri - PIQU) (Fig. 1).

Amostragens mensais foram realizadas durante o período de novembro de 1983 a outubro de 1989. Nas estações do reservatório de Itaipu (GUAI, SHEL e FOZ) as coletas foram realizadas até janeiro de 1989, enquanto que nas demais estações, estenderam-se até outubro de 1989, apresentando, entretanto, início nos períodos de outubro de 1985, nas estações de GUAC, OCOI, SFFA, JUSA e MONT, abril de 1986 em SFVE, setembro de 1986 em PIQU e abril de 1987 em IGUA. As coletas foram bimestrais apenas durante o primeiro semestre de 1987.

Os aparelhos de pesca, expostos por 24 horas, consistiram de redes de espera simples, com malhas de três a $16 \mathrm{~cm}$ entre nós opostos, redes de espera tresmalho (feiticeiras) de seis a $10 \mathrm{~cm}$ entre nós opostos e espinhéis com 30 e 50 anzóis.

A abundância de cada espécie amostrada foi determinada através da captura por unidade de esforço (CPUE) expressa em número de indivíduos capturados por $1000 \mathrm{~m}^{2}$ de rede em 24 horas de pesca.

Os impactos decorrentes da formação do reservatório de Itaipu sobre as populações de peixes foram avaliados através de comparações das freqüências de espécies coletadas entre novembro de 1987 e outubro de 1988, nas estações GUAC, OCOI, SFFA e SFVE e no trecho represado do rio Paraná, e as obtidas em investigações realizadas por CTSA/ITAIPU $(1979,1981)$ antes da formação do reservatório de Itaipu. 


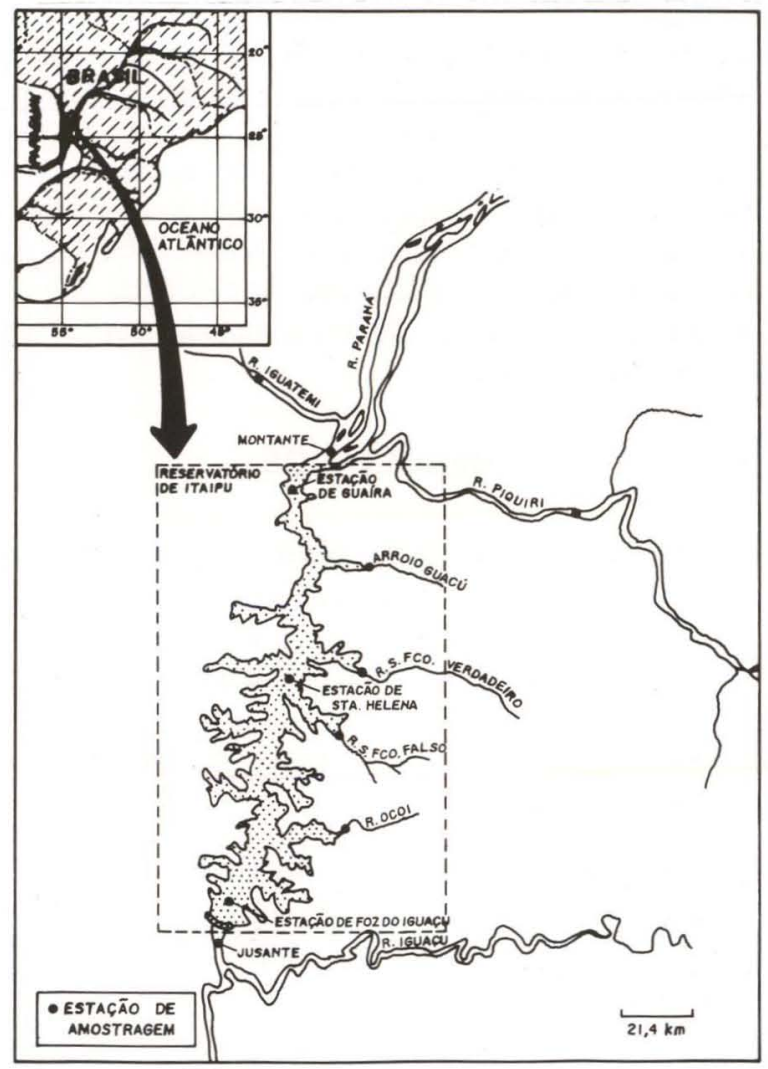

Fig. 1. Localização das estações de amostragem.

A análise da variação espacial e temporal da diversidade ictiofaunística (H') foi realizada com base nos resultados obtidos pelo índice de diversidade de Shannon-Wiener (PIELOU 1975), sendo a eqüitabilidade na distribuição das espécies avaliada como a razão deste índice e a diversidade máxima possível (H'máx). As expressões utilizadas foram:

$$
H^{\prime}=-\sum\left(\frac{n i}{N}\right) \times \log \left(\frac{n i}{N}\right) ; H^{\prime} \text { máx }=\log S
$$

onde: (ni) número de índivíduos da espécie "i”, (N) número total de indivíduos capturados e (S) número de espécies.

A riqueza de espécies (d) foi calculada com base na equação (ODUM 1985):

$$
d=\frac{S-1}{\log N}
$$

Na comparação entre as estações de coleta, realizada com base na abundância das espécies, em cada período de estudo, empregou-se o índice métrico de Canberra 
para a obtenção da matriz de distâncias e o agrupamento da associação média não ponderada (UPGMA) como método de ligação. Antes do cálculo da matriz de distâncias foi aplicada a transformação $\log (x+1)$, indicada na normalização de dados de abundância (SOKAL \& ROHLF 1995). Entre os critérios usados no julgamento da adequação do método utilizado na obtenção dos dendrogramas, analisaram-se os valores do coeficiente de correlação cofenética (CCC), ou seja, o coeficiente de correlação de Pearson calculado entre as distâncias originais dos pontos obtidos pela medida de semelhança e as novas medidas obtidas pelo método de ligação (EVERITT \& DUNN 1991).

\section{RESULTADOS}

\section{1) As populações de peixes antes e após o represamento}

A pesca experimental realizada antes do represamento no trecho que atualmente é ocupado pelo reservatório de Itaipu e em seus tributários da margem esquerda, revelaram como espécies abundantes, Hoplias aff. malabaricus, Ancistrus cirrhosus e Ageneiosus valenciennesi (Tab. I). O gênero Hypostomus, composto por várias espécies (não identificadas) foi, entretanto, o mais freqüente, contribuindo com quase a metade do total capturado.

Tabela I. Freqüência relativa das espécies coletadas antes (1978-81) e após (1987-88) a formação do reservatório de Itaipu. Rios Ocoí (OCOÍ), São Francisco Falso (SFFA), São Francisco Verdadeiro (SFVE), Arroio Guaçu (GUAC), (RES). Abundância: (+) menor que $5 \%$, (++) 5-10\%, (+++) 10-50\%, (++++) maior que $50 \%$.

\begin{tabular}{|c|c|c|c|c|c|}
\hline \multirow[b]{2}{*}{ Espécies } & \multicolumn{5}{|c|}{ Estaçōes de amostragem } \\
\hline & ocol & SFFA & SFVE & GUAC & RES \\
\hline & Antes Após & Antes Após & Antes Após & Antes Após & Antes Após \\
\hline
\end{tabular}

Hypostomus sp.

Hoplias aff. malabaricus (Block, 1794)

Ancistrus cirrhosus (Valenciennes, 1840)

Ageneiosus valenciennesi Bleeker, 1864

Rhamdia hilarii Valenciennes, 1840

Astyanax eigenmanniorum (Cope, 1864)

Oligosarcus sp.

Plagioscion squamosissimus (Heckel, 1840)

Gymnotus carapo Linnaeus, 1879

Galeocharax knerii (Steindachner, 1879)

Geophagus brasiliensis (Quoy \& Gamard, 1824)

Prochilodus lineatus Valenciennes, 1836)

Salminus maxillosus Valenciennes, 1840

Cyprinus carpio Linnaeus, 1758

Rhinodoras dorbignyi Kröyer, 1855

Trachydoras paraguayensis (Elgenmann \& Ward, 1907)

Catathyridium jenynsii (Gunther, 1862)

Crenicichla niederleinii (Holmberg, 1891)

Hemisorubim platyrhynchos (Valenciennes, 1840)

Loricaniichthys sp.

Astyanax bimaculatus (Linnaeus, 1758)

Serrasalmus marginatus Valenciennes, 1847

Astyanax cf. scabripinnis Elgenmann, 1927

Eigenmannia virescens (Valenciennes, 1847)

\begin{tabular}{|c|c|c|c|c|c|c|c|c|c|}
\hline+++ & + & ++++ & + & ++++ & + & & + & +++ & + \\
\hline+ & + & +++ & + & +++ & + & +++ & + & + & + \\
\hline \multirow[t]{2}{*}{+++} & & + & & + & & + & & + & \\
\hline & & + & & & + & & & +++ & + \\
\hline+ & & + & & + & & +++ & & & \\
\hline+++ & & + & & + & & & & + & \\
\hline \multirow[t]{2}{*}{+} & & + & & & & +++ & & + & \\
\hline & + & & + & & + & & + & + & + \\
\hline \multirow[t]{2}{*}{+} & & + & + & + & & & + & & + \\
\hline & + & & & + & & & + & + & + \\
\hline+ & & + & & & & + & & & \\
\hline \multirow[t]{6}{*}{+} & & & & & & & & + & + \\
\hline & & & & & & & & + & + \\
\hline & + & & & + & & + & & & \\
\hline & + & & + & & + & & + & + & + \\
\hline & +++ & & + & & +++ & & & + & + \\
\hline & + & & & & & & & + & \\
\hline \multirow[t]{2}{*}{+} & + & & + & & & & + & + & + \\
\hline & +++++ & & +++ & & +++ & & + & + & + \\
\hline \multirow[t]{3}{*}{+} & + & & + & & & & + & + & + \\
\hline & + & & + & & + & & + & + & + \\
\hline & + & & & + & + & & + & $\begin{array}{l}+ \\
+\end{array}$ & + \\
\hline
\end{tabular}


Tabela I. Continuação.

\begin{tabular}{|c|c|c|c|c|c|c|c|}
\hline \multirow{3}{*}{ Espécies } & \multicolumn{7}{|c|}{ Estações de amostragem } \\
\hline & \multicolumn{2}{|c|}{ ocol } & \multirow{2}{*}{$\frac{\text { SFFA }}{\text { Antes Após }}$} & SFVE & GUAC & \multicolumn{2}{|c|}{ RES } \\
\hline & Antes & Após & & Antes Após & Antes Após & Antes & sApós \\
\hline Leporinus lacustris Campos, 1945 & + & & & & & + & \\
\hline Pimelodus maculatus Lacépède, 1803 & & + & + & + & + & + & + \\
\hline Brycon orbignyanus (Valenciennes, 1849) & & & & & + & + & + \\
\hline Potamotrygon motoro (Müller \& Henle, 1841) & & & & & & + & \\
\hline Megalancistrus aculeatus (Perugia, 1891) & & & & & & + & \\
\hline Schizodon altoparanae Garavello \& Britski, 1990 & & & & & & + & + \\
\hline Steindachneridion $\mathrm{sp}$. & & & & & & + & \\
\hline Cichlasoma paranaense Kullander, 1983 & + & & & & + & & \\
\hline Astyanax fasciatus (Cuvier, 1819) & + & & & & + & & \\
\hline Brycon iheringi (Boulenger, 1887) & + & & & & & & \\
\hline Crenicichla haroldoi Luengo \& Britsld, 1974 & & & & & + & & \\
\hline Leporellus vittatus (Valenciennes, 1849) & & & & + & & + & + \\
\hline Leporinus obtusidens (Valenciennes, 1847) & & & & + & + & + & + \\
\hline Leporinus striatus Kner, 1859 & & & & & & + & \\
\hline Parauchenipterus galeatus (Linnaeus, 1766) & & + & + & + & + & + & + \\
\hline Paulicea luetkeni (Steindachner, 1875) & & & & & & + & + \\
\hline Pimelodella sp. & & + & & + & + & & + \\
\hline Pimelodella gracilis (Valenciennes, 1840) & & & & & & + & \\
\hline Pimelodus cf. blochi (Valenciennes, 1840) & & & & & & + & \\
\hline Pimelodus fur (Reinhardt, 1874) & & & & & & + & \\
\hline Pseudocetopsis gobioides (Kner, 1857) & & & & & & + & \\
\hline Pseudopimelodus zungaro (Humboldt, 1833) & + & & & & & & \\
\hline Pseudoplatystoma corruscans (Agassiz, 1829) & & & & + & + & + & + \\
\hline Serrasalmus spilopleura Kner, 1860 & & & + & & + & + & \\
\hline Sternopygus macrurus (Bloch \& Schneider, 1801) & & & & & & + & + \\
\hline Auchenipterus nuchalis (Spix, 1829) & & + & + & +++ & + & & +++ \\
\hline Hypophthalmus edentatus (Spix, 1829) & & + & & + & & & +++ \\
\hline Steindachnerina insculpta Fernandez-Yepez, 1948 & & +++ & +++ & + & +++ & & + \\
\hline Roeboides paranensis Pignalberi, 1975 & & + & + & & + & & + \\
\hline Loricaria sp. & & + & + & + & + & & + \\
\hline Iheringichthys labrosus (Kröyer, 1874) & & + & + & + & + & & + \\
\hline Ageneiosus ucayalensis Castelnau, 1875 & & & + & + & + & & + \\
\hline Rhaphiodon vulpinus Agassiz, 1829 & & + & + & + & + & & + \\
\hline Loricariichthys platymetopon Isbrücker \& Nijssen, 1979 & & & & & + & & + \\
\hline Schizodon borellii (Boulenger, 1875) & & & & & + & & + \\
\hline Apteronotus sp. & & + & + & + & + & & + \\
\hline Pterodoras granulosus (Valenciennes, 1873) & & + & + & + & + & & + \\
\hline Eigenmannia sp. & & & + & + & + & & + \\
\hline Acestrorhynchus lacustris (Reinhardt, 1874) & & + & + & + & & & + \\
\hline Pinirampus pirinampu (Spix, 1829) & & & & & & & + \\
\hline Doras eigenmanni (Boulenger, 1825) & & + & & + & & & + \\
\hline Leporinus friderici (Bloch, 1794) & & & & & + & & + \\
\hline Crenicichla britskii Kullander, 1982 & & & & + & + & & + \\
\hline Hoplosternum littorale (Hancock, 1828) & & & & & & & + \\
\hline Apareiodon affinis (Steindachner, 1879) & & & & & + & & + \\
\hline Eigenmannia trilineata (Lopez \& Castello, 1966) & & & & & + & & \\
\hline Parodon tortuosus Eigenmann \& Norris, 1900 & & & & + & + & & \\
\hline Satanoperca pappaterra (Heckel, 1810) & & & & & & & + \\
\hline Leporinus octofasciatus Steindachner, 1917 & & & + & & + & . & + \\
\hline Apteronotus albifrons (Linnaeus, 1766) & & + & & + & & & + \\
\hline Ancistrus sp. & & + & & & & & \\
\hline Pimelodus sp. & & & + & & & & + \\
\hline Rhamphichthys rostratus (Linaeus, 1766) & & + & + & + & & & + \\
\hline Leporinus elongatus Valenciennes, 1849 & & & + & & + & & + \\
\hline Sorubim cf. lima (Schneider, 1801) & & & & & & & + \\
\hline Loricaria prolixa Isbrücker \& Nijssen, 1978 & & & & & + & & \\
\hline Moenkhausia intermedia Eigenmann, 1908 & & & & & & & + \\
\hline Pimelodus ornatus Kner, 1857 & & & + & & + & & + \\
\hline Rhinelepis aspera Agassiz, 1828 & & & & & & & + \\
\hline Rhamdia spp. & & & + & & & & \\
\hline
\end{tabular}


Cinco anos após a formação do reservatório de Itaipu, duas espécies (Auchenipterus nuchalis, insetívora, e Hypophthalmus edentatus, planctófaga), bastante esporádicas na pesca experimental realizada anteriormente, constituíram $50 \%$ das capturas totais neste ambiente. Nos tributários, este predomínio coube a Loricariichthys sp. e a Steindachnerina insculpta, sendo que, anteriormente, a primeira foi registrada apenas no rio Paraná, e a segunda foi rara.

Além de Loricariichthys sp., outras espécies anteriormente restritas às capturas no rio Paraná ocuparam de forma eficiente os rios laterais após a formação do reservatório. São elas: Plagioscion squamosissimus, Trachydoras paraguayensis, Astyanax bimaculatus, Eigenmannia virescens, Pimelodus maculatus e Parauchenipterus galeatus.

No geral, o número de espécies duplicou em todos os tributários do reservatório em relação ao período de pré-represamento, com uma pronunciada substituição de espécies.

\section{2) Diversidade, eqüitabilidade e riqueza de espécies}

A diversidade elevou-se desde a formação do reservatório, em todas as estações amostradas, exceto aquelas do corpo do reservatório que, a despeito dos altos valores iniciais, mostrou decréscimo gradativo após o quarto ano de sua formação. No geral, os valores de diversidade do reservatório de Itaipu foram superiores àqueles do segmento do rio Paraná antes do represamento (Fig. 2).

A comparação da diversidade ictiofaunística, antes e após o represamento, mostra que esta se elevou nos tributários (GUAC, OCOÍ, SFFA, SFVE) após a formação do reservatório, como resultado da já referida entrada de espécies antes restritas às amostragens no rio Paraná. $\mathrm{O}$ aumento da diversidade ocorreu, entretanto, após o transcurso de algum tempo, sendo este período menor para o SFFA. Os valores da diversidade específica no reservatório, quando comparados com aqueles do rio Paraná, antes do represamento, foram maiores até 1987. Entre as estações situadas no corpo principal do reservatório, FOZ, a mais lacustre, exibiu a menor diversidade ictiofaunística acompanhada da menor riqueza e eqüitabilidade de espécies.

A estação de amostragem de MONT, seguida de IGUA e JUSA foram as que apresentaram os maiores índices de diversidade de Shannon-Wiener $(4,87,4,82$, 4,72 , respectivamente), e a maior eqüitabilidade na distribuição dos indivíduos capturados entre as espécies. Baixos valores de diversidade específica foram registrados em SFVE e OCOÍ.

A riqueza de espécies foi, entretanto, maior no reservatório de Itaipu, sendo relevante a contribuição de GUAI. Por outro lado, os menores valores obtidos para a riqueza foram constatados nos tributários OCOÍ, SFVE e SFFA.

\section{3) Similaridade Ictiofaunística}

As similaridades entre as estações de amostragem em relação à composição e abundância das espécies durante o período de 1983-89 são mostradas na figura 3 . $\mathrm{O}$ dendrograma de distâncias permite caracterizar, nas proximidades do nível 0,4, cinco ambientes distintos: (1) a área do reservatório de Itaipu; (2) seus tributários da margem esquerda; (3) MONT e IGUA; (4) JUSA; e (5) PIQU. 


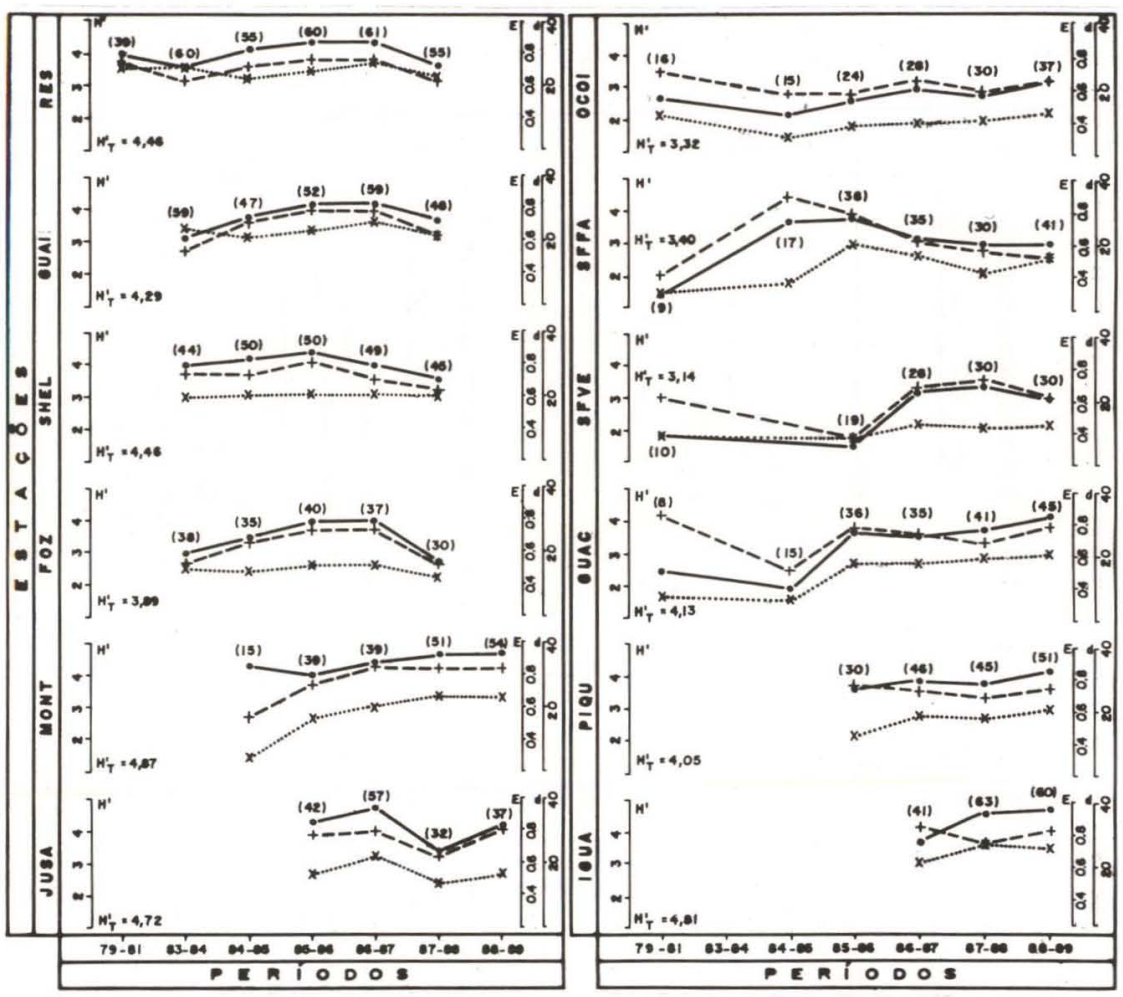

Fig. 2. Valores do indice de diversidade de Shannon-Wiener $\left(\mathbf{H}^{\prime}\right.$ - linha continua), da equitabilidade de espécies ( $E$ - linha interrompida), da riqueza de espécies (d - linha pontilhada) e do número de espécies (entre parênteses) obtidos por período de estudo em cada estação de amostragem $\left(\mathrm{H}_{\mathrm{t}}=\right.$ = diversidade específica para todo o período de estudo).

Verifica-se que as distâncias entre os grupos foram gradativamente menores com o decorrer do tempo, revelando uma tendência de crescente uniformidade ictiofaunística na região. JUSA e PIQU, entretanto, foram estações que apresentaram ictiofauna pouco similar às demais. A jusante do reservatório de Itaipu observou-se a presença de espécies exclusivas ao trecho médio do rio Paraná. No PIQU observou-se também exclusividade para as espécies de Astyanax cf. marionae Eigenmann, 1911, Apareiodon piracicabae Elgenmann, 1907, Leporinus amblyrhynchus Britski \& Garavello, 1987, Myloplus sp., Oligosarcus sp., Pimelodus paranaensisBritski \& Lajeani, 1988 e Steindachneridion sp. e ausência de famílias como Sciaenidae, Doradidae, Cynodontidae e Serrasalmidae.

\section{DISCUSSÃO}

\section{1) As populações de peixes antes e após o represamento}

As alterações na ictiofauna regional, impostas pelo represamento de Itaipu, compreenderam grandes modificações na composição da assembléia e a virtual 

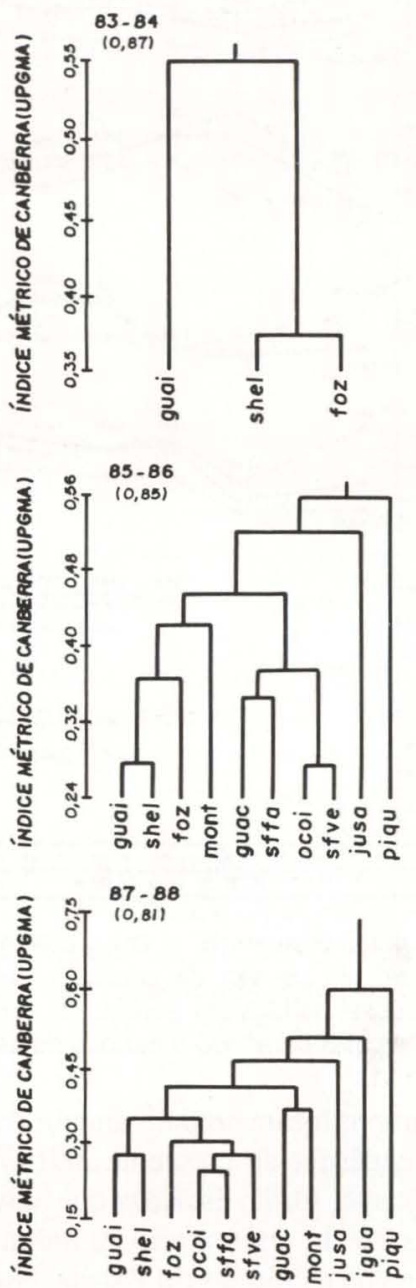
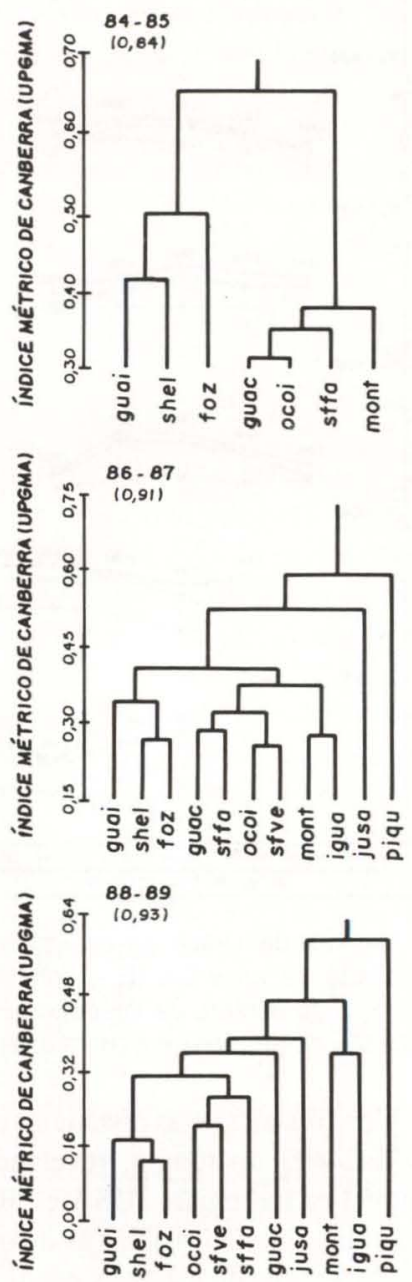

Fig. 3. Dendrogramas resultantes das análises de agrupamento sobre a matriz de abundância de espécies nas estações de amostragem, utilizando o índice métrico de Canberra e a UPGMA, como método de ligação, para cada periodo de estudo (entre parênteses: valores dos coeficientes de correlação cofenética).

extinção local de alguns de seus elementos. As espécies dominantes, antes do represamento, tiveram suas participações nas capturas no reservatório drasticamente reduzidas no período que se seguiu. O caráter reofilico dos cascudos (Hypostomus spp. e A. cirrhosus), anteriormente registrados, explicam pelo menos em parte, suas baixas freqüências na área represada. Hoplias aff. malabaricus com previsões 
iniciais de se manter dominante no reservatório (CTSA/ITAIPU 1979), não foi bem sucedida nos primeiros anos de colonização. O desenvolvimento de áreas litorâneas, a escassez de macrófitas aquáticas e as flutuações de nível da água que caracterizam este período devem se relacionar a este fato. Algumas espécies como Otocinclus vittatus Regan, 1904, Xenurobrycon macropus Myers \& Ribeiro, 1945, Tetragonopterus argenteus, Roeboides prognatus (Boulenger, 1895), presentes na fase rio, não foram capturadas na região nos seis primeiros anos do reservatório. Todas elas, no entanto, eram esporádicas anteriormente.

Plagioscion squamosissimus, um carnívoro introduzido na bacia antes do represamento (TORLONI et al. 1993) e presente nas amostragens realizadas no rio Paraná naquele período, destacou-se como uma espécie bem sucedida na colonização do reservatório. Esta espécie está entre as dominantes nas capturas comerciais de todos os grandes reservatórios da bacia do rio Paraná (TORLONI et al. 1994; AgostinHo 1994). Prochilodus lineatus, supostamente ameaçada pelas novas condições (CTSA/TTAIPU 1979), teve participação relevante após o represamento. AGostinHo et al. (1993) atribuem este fato, pelo menos em parte, à existência de uma vasta planície de inundação no trecho contíguo do rio Paraná, a montante, onde a espécie tem seu desenvolvimento inicial.

Algumas espécies, além de serem sensíveis à mudança na dinâmica da água, têm sua sobrevivência ameaçada pela escassez de alimento alóctone, esperada pela redução imposta pelo represamento na proporção entre as áreas terrestres com vegetação e a lâmina de água. Este deve ser o caso de espécies como Brycon orbignyanus e Piaractus mesopotamicus (Holmberg, 1887), que tiveram suas ocorrências registradas apenas nos primeiros dois anos após o represamento, embora freqüentes acima e abaixo do reservatório durante todo o período.

Hypophthalmus edentatus, uma espécie zooplanctófaga filtradora, até então ausente nos trechos superiores da bacia do rio Paraná, situou-se entre as três mais abundantes durante todo o período de estudos após o represamento. Esporádica na fase rio, com apenas três exemplares capturados, sua dominância na assembléia se evidenciou já no primeiro ano da formação do reservatório. AGOSTINHO et al. (1994) levantam três possíveis causas para este fato: (1) o estoque teria sido recrutado como adulto a partir de áreas lacustres, restritas na bacia, não identificadas e não amostradas antes do represamento; ou (2) o recrutamento seria a partir de migrações ascendentes de cardumes excepcionalmente grandes, no período imediatamente anterior ao barramento, e provenientes de áreas alagáveis dos segmentos inferiores; ou ainda (3) teriam origem no próprio reservatório a partir de uma reprodução muito bem sucedida imediatamente após o represamento, um crescimento numa taxa elevadíssima e acompanhada de uma maturação precoce, durante o primeiro ano. A última hipótese é suportada pelo caráter oportunista que a espécie apresentou ao responder prontamente de forma positiva às alterações ambientais supostamente benéficas, reproduzindo-se durante quase todo o segundo ano e apresentando dois picos de desova, quando o normal para seu ciclo reprodutivo é uma sazonalidade bem pronunciada (BENEDITO 1989).

Segundo AGOSTINHO et al. (1992), a localização do reservatório de Itaipu, e suas características constituíram-se em fatores atenuantes ao impacto do represamento. $\mathrm{O}$ fato de a barragem ter sido construída a aproximadamente $150 \mathrm{~km}$ abaixo 
das Sete Quedas de Guaíra, uma barreira natural que limitava a distribuição das populações do médio Paraná, representou a subtração de um trecho relativamente pequeno e limítrofe da área de distribuição das espécies. Tal localização provocou, entretanto, a submersão da barreira natural, o que possibilitou a introdução a montante de mais de 15 espécies do médio e baixo Paraná. O curto tempo médio de residência da água (40 dias) e a velocidade relativamente alta $(0,6 \mathrm{~m} / \mathrm{s}$ na superfície), por outro lado, parece viabilizar a orientação das espécies migradoras que habitam atualmente seu terço fluvial.

Após sete anos da formação do reservatório de Itaipu, os indicadores de instabilidade, como alterações na abundância, substituições de espécies e predomínio daquelas com características r-estrategistas, peculiares à fase inicial do represamento, ainda predominaram. A instabilidade ambiental decorrente de variações de nível que caracterizaram os três primeiros anos, e as interações ensejadas pela elevada diversidade devem explicar as variações.

O recrutamento dos estoques no reservatório é controlado, em grande parte, pelos processos vigentes na planície alagável do rio Paraná a montante, que têm como principal função de força o regime de cheias, este regulado aleatoriamente pelas barragens localizadas nos trechos superiores da bacia (AGOSTINHO \& ZALEWSKI 1995; GOMES \& AGOSTINHO no prelo). As variações anuais na intensidade, duração e época das cheias leva a flutuações populacionais marcantes na abundância relativa das espécies, favorecendo algumas e sendo restritivas a outras (AGOSTINHO et al. no prelo) e influenciando a composição da ictiofauna no reservatório.

\section{2) Diversidade, eqüitabilidade e riqueza de espécies}

Após a formação do reservatório, a diversidade ictiofaunística foi superior àquela do período pré-represamento para os tributários do reservatório, em decorrência do ingresso de espécies provenientes de outras áreas, as quais anteriormente tinham dificuldade na transposição de obstáculos naturais (saltos, estreitos). Na área do reservatório, por outro lado, restrições impostas à assembléia lótica original (condições inadequadas para o desenvolvimento e reprodução) e o sucesso de espécies oportunistas, conduziram ao aumento da diversidade apenas nos primeiros anos de formação do reservatório, numa proporção inferior à constatada para seus tributários.

Ao longo do corpo principal do reservatório de Itaipu, a estação de SHEL, zona de transição, exibiu a maior diversidade de espécies, seguida por GUAI, zona fluvial. A primeira caracteriza-se pela abundante quantidade de vegetação semi-submersa e maior proporção de vegetação aquática flutuante, o que incrementa a disponibilidade de abrigo e alimento aos jovens, além de constituir-se por locais propícios a refúgio de presas. Além disso, a esta região confluem grandes tributários em ambas as margens. A estação de GUAI, com características semi-lóticas, permite a permanência de espécies de habitats lóticos e lênticos. FOZ, por outro lado, apresenta o trecho mais lêntico, mais profundo, com grande influência da operação da barragem e mais pobre em nichos exploráveis, correspondendo ao local de menor diversidade específica. FERREIRA (1984) também encontrou valores decrescentes a partir da estação mais afastada de Curuá-Una à mais próxima. 
Em geral, a diversidade do reservatório foi sensivelmente superior a de seus tributários da margem esquerda. Esta menor diversidade provavelmente esteja relacionada à pequena área destas sub-bacias (WоOTON 1990).

Os segmentos do rio Paraná a montante e a jusante do reservatório revelaram, entretanto, diversidade marcadamente superior àquela do corpo principal do reservatório. No primeiro caso é relevante a presença da planície de inundação que contribui com uma grande variedade de biótopos, além de contar com a afluência dos grandes rios da região. Além disso, a dispersão de pelo menos 15 espécies, antes confinadas aos trechos médios e inferiores, para o alto rio Paraná, contribuiu, pelo menos transitoriamente, para o aumento da riqueza específica. No segundo, a concentração de peixes em migração ascendente ou atraídos por condições alimentares excepcionais (outros peixes, plâncton oriundos do reservatório, insetos atraídos pelas luzes do canteiro de obras e da cidade de Foz do Iguaçu) parece decisiva para este quadro. Ressalta-se que a instabilidade dos trechos a jusante de barragens, decorrente de pulsos de vazão e da qualidade da água evertida, o acúmulo de predadores, a falta de áreas de refúgio e alimentação para jovens e espécies forrageiras, além da inadequação para a desova são fatores que certamente se opõem a altas diversidades (MERONA et al. 1987; AGOSTINHO et al. 1992; GoDINHO 1993).

Entre todos os ambientes estudados, os valores do índice de diversidade, foram maiores e crescentes em IGUA, que apresenta como característica uma ampla planície alagável, com curso meandrante e grande variedade de biótopos.

Vários pesquisadores como EMERY (1978), RAHEL et al. (1984), GARUTTI (1988), MINNS (1989), JACKSON \& HARVEY (1989) admitem que os fatores responsáveis pela caracterização na riqueza de espécies, área do habitat e a heterogeneidade espacial revelam-se como características associadas positivamente. A área da bacia a montante parece não ter sido relevante, pelo menos na escala utilizada, para explicar os valores estimados da diversidade. Assim, GUAC apresentou a maior riqueza de espécies, sendo superior até mesmo àquela do rio Piquiri, com bacia consideravelmente maior. Influência particular sobre a riqueza específica, no presente estudo, foi a proximidade de planícies alagáveis, como demonstram os valores obtidos nas estações MONT, GUAI no próprio reservatório, e mesmo do GUAC, que é o último tributário a montante do reservatório.

Os índices de diversidade, eqüitabilidade e riqueza de espécies, apresentaram tendências semelhantes entre os períodos de estudo na maioria das estações amostradas, constituindo-se, portanto, em parâmetros similares quanto à eficiência na avaliação da ordem (ou desordem) no sistema.

\section{3) Similaridade ictiofaunística}

A crescente similaridade entre as populações de peixes das diferentes estações de amostragem, com o decorrer do tempo, pode ser explicada pela dispersão de espécies na região, a partir do reservatório, evidenciada pelo aumento no número de espécies nos tributários deste, a sua montante e no rio Iguatemi.

A semelhança entre as estações do corpo principal do reservatório, em todos os períodos, é fato esperado, uma vez que são estações com características ambientais similares e sujeitas às perturbações também semelhantes. 
Outros dois grupos de estações com características bastante semelhantes foram as dos tributários do reservatório e as do segmento a montante e rio Iguatemi. Esse padrão, entretanto, não foi constante ao longo dos períodos estudados, revelando o caráter gradativo da dispersão.

JUSA e PIQU corresponderam aos ambientes mais distintos dentre os amostrados. Segundo AgostinHo \& Júlio (no prelo), no trecho do rio Paraná a jusante do reservatório de Itaipu registram-se 16 espécies típicas dos segmentos inferiores da bacia e não capturadas na represa ou no alto Paraná. Algumas delas destacam-se pelo caráter anádromo e outras pela presença marcante na bacia do Paraguai. Quanto a PIQU, ambiente com corredeiras, saltos, estreitos e forte declividade, segundo AGOSTINHO \& JÚLIO (no prelo), caracteriza-se pela ausência de muitas espécies, gêneros e mesmo algumas famílias como Ageneiosidae, Doradidae, Cynodontidae e Sciaenidae, com ampla distribuição na bacia e abundância de outras, esporádicas nos demais corpos de água da região.

Em síntese a formação do reservatório de Itaipu conduziu a alterações na abundância de espécies e na estrutura da ictiofauna de toda sua área de influência: espécies oportunistas obtiveram sucesso na exploração dos recursos disponíveis, enquanto outras, características de ambientes lóticos, tiveram suas abundâncias reduzidas ou mesmo não foram registradas nas amostragens. Nos tributários da margem esquerda e corpo principal do reservatório de Itaipu, em geral, a diversidade ictiofaunística foi superior aquela do período pré-represamento, sendo os menores valores dos índices de diversidade, eqüitabilidade e riqueza de espécies registrados para o segmento do reservatório mais próximo à barragem. Dentre todos os ambientes estudados, a diversidade específica foi maior para Montante, Iguatemi e Jusante, respectivamente. As estações de amostragem foram agrupadas em cinco áreas de maior semelhança com base na composição e abundância das espécies: (1) corpo principal do reservatório de Itaipu; (2) tributários do reservatório; (3) Montante e Iguatemi; (4) Jusante; e (5) Piquiri.

AGRADECIMENTOS. Ao Núcleo de Pesquisas em Limnologia, Ictiologia e Aqüicultura, pelo apoio logístico. À Itaipu Binacional pelo apoio financeiro. Ao Prof. Luiz Carlos Gomes e ao Prof. Dr. Sidinei Magela Thomaz pela correção do Abstract. Ao Jaime Lopes Pereira pela confecção dos gráficos. À bibliotecária Maria Salete Ribelatto Arita pelos serviços bibliográfícos.

\section{REFERÊNCIAS BIBLIOGRÁFICAS}

AGostinHo, A.A. 1994. Considerações sobre a atuação do setor elétrico na preservação da fauna aquática e dos recursos pesqueiros. In: COMASE/ELETROBRÁS (Ed.). Seminário sobre fauna aquática e o setor elétrico brasileiro - Caderno IV: Estudos e Levantamentos. Rio de Janeiro, Comase/Eletrobrás, $86 \mathrm{p}$.

Agostinho, A.A. \& M. ZALEWSKI. 1995. The dependence of fish community structure and dynamics on floodplain and riparian ecotone zone in Paraná River, Brazil. Hydrobiologia 303: 141-148. 
Agostinho, A.A.; H.F. Julio JR. \& J.R. BorghetTI. 1992. Considerações sobre os impactos dos represamentos na ictiofauna e medidas para sua atenuação. Um estudo de caso: Reservatório de Itaipu. Rev. Unimar 14 (supl.): 89-107.

Agostinho, A.A.; A.E.A.M. Vazzoler; L.C. Gomes \& E.K. OKadA. 1993. Estratificación espacial y comportamiento de Prochilodus scrofa em distintas fases del ciclo de vida, en la planicie de inundación del alto rio Paraná y embalse de Itaipu, Paraná, Brasil. Rev. Hydrobiol. trop. 26 (1): 79-90.

Agostinho, A.A.; E. Benedito-Cecilio; L.C. Gomes \& A.A. Sampaio. 1994. Spatial and temporal distribution of sardela, Hypophthalmus edentatus (Pisces, Siluroidei), in the area of influence of the Itaipu reservoir (Paraná, Brazil). Rev. Unimar 16 (supl. 3): 27-40.

Agostinho, A.A.; A.E.A.M. Vazzoler \& S.M. Thomaz. 1995. The high river Paraná basin: limnological and icthtiological aspects, p. 61-103p. In: J.G. TUNDISI \& T.M. TUNDISI. Limnology in Brazil. Rio de Janeiro, ABC/SBL, 376p.

Agostinho, A.A. \& H.F. Julio JR. (no prelo). Peixes da bacia do alto rio Paraná. In: R.L. MCCONNELL (Ed.). Ecologia de comunidades de peixes tropicais. São Paulo, EDUSP.

Agostinho, A.A.; L.C. Gomes \& M. ZALEWSKI. (no prelo). The importance of floodplains for the dynamics of fish communities of the upper river Paraná. In: M. ZALEWSKI \& J. THORPE. Fish and land/inland water ecotones. Paris, Unesco MAB Series.

BENEDITO, E. 1989. Estrutura da população, reprodução e seletividade amostral de Hypophthalmus edentatus (Spix, 1829)(Osteichthyes, Siluriformes) no reservatório de Itaipu - PR. Tese de Mestrado, não publicada, Curso de Pós-Graduação em Zoologia, Universidade Federal do Paraná, Curitiba, 237p.

CTSA/ITAIPU (Companhia de Tecnologia de Saneamento Ambiental/Itaipu Binacional). 1979. Itaipu Binacional -Ictiofauna. Curitiba, CETESB, 126p.

ـ 1981. Itaipu Binacional-Ictiofauna. Complementação do Inventário Ictiofaunístico. Curitiba, CETESB, vol. 3.

EMERY, A.R. 1978. The basis of fish community structures marine and freshwater comparisons. Env. Biol. Fish. 3 (1): 33-47.

EveritT, B. \& G. DunN. 1991. Applied multivariate data analysis. Kent Edward Arnold, 304p.

FERREIRA, E.J.G. 1984. A ictiofauna da represa hidrelétrica de Curuá-Una, Santarém, Pará. I. Lista e distribuição das espécies. Amazoniana 8 (3): 351-363.

GARUTTI, V. 1988. Distribuição longitudinal da ictiofauna em um córrego da região noroeste do estado de São Paulo, Bacia do rio Paraná. Rev. Brasil. Biol. 48 (4): 747-759.

GodinHo, A.L. 1993. E os peixes de Minas em 2010? Ciência Hoje 16 (9): 44-49.

GoMES, L.C. \& A.A. AGostinHo. (no prelo). Influence of the flood regime on the nutritional state and juvenile recruitment of Prochilodus scrofa Steindachner in Upper Paraná River, Brazil. Fisheries Management and Ecology. 
JACKSON, D.A. \& H.H. HARVEY. 1989. Biogeographic associations in fish assemblages: local vs. regional processes. Ecology 70 (5): 1472-1484.

Merona, B. DE. 1986. Aspectos ecológicos da ictiofauna no Baixo Tocantins. Acta Amazonica 16/17: 109-124 [1987].

Merona, B., J.L. Carvalho \& M.M. BitTencourT. 1987. Les effets immédiats de la fermeture du barrage de Tucurui (Brésil) sur l'ichtyofaune en aval. Rev. Hydrobiol. Trop. 20 (1): 73-84.

MinNS, C.K. 1989. Factors affecting fish species richness in Ontario Lakes. Trans. Amer. Fish. Soc. 118: 533-545.

ODUM, E.P. 1985. Ecologia. Rio de Janeiro, Interamericana, 434p.

PIELOU, E.C. 1975. Ecological Diversity. New York, J. Wiley, 165p.

RAHEL, F.J.; J.D. LYONS \& P.A. COCHRAN. 1984. Stochastic or deterministic regulation of assemblage structure? It may depend on how the assemblage is defined. Amer. Nat. 124: 583-589.

SoKal, R.R. \& F.J. RoHLF. 1995. Biometry. The Principles and Practice of Statistics in Biological Research. 3nd. ed. New York, W.H. Freeman, $3^{\text {nd }}$ ed., $887 \mathrm{p}$.

Torloni, C.E.C.; J.J. Santos; A.A.C. Carvalho JR. \& A.R.A. CôRrea. 1993. A pescada do piauí Plagioscion squamosissimus (Heckel, 1840)(Osteichthyes, Perciformes) nos reservatórios da Companhia Energética de São Paulo CESP. São Paulo, CESP, 23p.

Torloni, C.E.C.; A.R.A. Côrrea; A.A.C. Carvalho JR.; J.J. SANTOS; J.L. GonÇAlves; E.J. Gereto; J.A. CruZ; J.A. Moreira; D.C. Silva; E.F. DeUS \& A.S. FERREIRA. 1994. Produção pesqueira e composição das capturas em reservatórios sob concessão da CESP nos rios Tietê, Paraná e Grande, no período de 1986 a 1991. São Paulo, CESP, 73p.

WETZEL, K.G. 1990. Reservoir ecosystems: conclusion and speculations, p. 227-238. In: K.W. Thornton; B.L. Kimmel \& F.E. Payne. Reservoir limnology ecological perspectives. London, John Wiley \& Sons Inc., 246p. Wootton, R.J. 1990. Ecology of teleost fishes. London, Chapman \& Hall, 440p.

Recebido em 21.VI.1996; aceito em 13.II.1997. 